\title{
Para Kenmotsu Manifoldun Skew Semi İnvaryant Altmanifoldları
}

\author{
Skew Semi Invariant Submanifolds of Para Kenmotsu Manifold
}

\author{
Ramazan SARI $^{* 1, a}$, İnan ÜNAL ${ }^{2, b}$, Elif AKSOY SARI ${ }^{3, c}$ \\ ${ }^{1}$ Gümüşhacıköy Hasan Duman Vocational Schools, Amasya University, Amasya, TURKEY \\ ${ }^{2}$ Department of Computer Engineering, Munzur University, Tunceli, TURKEY \\ ${ }^{3}$ Merzifon Vocational Schools, Amasya University, Amasya, TURKEY
}

•Geliş tarihi / Received: 03.09.2018 • Düzeltilerek geliş tarihi / Received in revised form: 09.11.2018 • Kabul tarihi / Accepted: 15.11 .2018

\section{Öz}

Bu çalışmada para Kenmotsu manifoldlaırn skew semi invaryant altmanifoldları çalışıldı. Bir örnek verildi ve distribüsyonların integrallenebilirlik şartları elde edildi. Para Kenmotsu space formun bu tür altmanifoldları incelendi ve bazı eğrilik özellikleri elde edildi. Son olarak para Kenmotsu manifoldların bir total geoedezik skew semi invaryant altmanifoldunun $\eta$-Einstein olduğu ispatlandı.

Anahtar kelimeler: Para Kenmotsu manifold, Para Kenmotsu uzay form, Skew semi invariant altmanifold

\begin{abstract}
In this paper skew semi invariant submanifolds of para Kenmotsu manifold are studied. An example is given and integrability conditions of distributions are obtained. This kind of submanifolds of para Kenmotsu space form are examined and some curvature properties are obtained. Finally it is proved that a totally geodesic skew semi invariant submanifold of para Kenmotsu manifold is $\eta$-Einstein.
\end{abstract}

Keyword: Para Kenmotsu manifold, Para Kenmotsu space form, Skew semi invariant submanifolds

*aa Ramazan SARI; ramazan.sari@ amasya.edu.tr; Tel: (0536) 43164 30; orcid.org/0000-0002-4618-8243 


\section{Introduction}

Para complex geometry is the geometry which is related to the algebra of para complex numbers (Cruceanu et al, 1996). The para complex structures is defined on a smooth manifold $M$ with the endomorphism $J: \Gamma(T M) \rightarrow \Gamma(T M), J^{2}=I$ such that the 1-eigen distributions are integrable and has the same dimension. There are some differences between para complex geometry and complex geometry. The product of two manifolds $M_{+} \times M_{-}$of the same dimension is the natural example of para complex manifold. These manifolds also have some applications in physics, they are related to supersymmetric field theories with Euclidean space time (Zamkovoy, 2009).

In 1985 Willams and Kaneyuki studied the almost para contact structure on $(2 n+1)$-dimensional pseudo-Riemannian manifold $M$ and showed the almost paracomplex structure on the product manifold of $M$ and real line $\mathbb{R}$ (Kaneyuki and Willams, 1985). Also Zamkovoy (Zamkovoy, 2009) studied an almost paracontact metric manifold and their subclasses. Similar to contact manifolds some classes of para contact manifolds are defined and this notion studied by geometers. One of them is para Kenmotsu manifolds and we focused on this notion in this paper.

The almost semi invariant submanifold of a Sasakian manifold was studied by Bejancu and Papaghuic (Bejancu and Papaghuic, 1984). They also gave a classification for submanifold classes of a Sasakian manifold. Ronsse defined skew CRsubmanifolds of Kaehler manifold, such a generalization of bi slant submanifold (Ronsse, 1990). After this notion is studied by several authors (Lui and Shao, 1999; Şahin, 2010).

Our aim in the present work is to extend the study of skew semi invariant submanifolds to the setting of a para Kenmotsu manifold. After giving fundamental facts about para Kenmotsu manifolds we examine the submanifolds of them. We recall the definition of skew semi invariant submanifold and we give an example. Morover we obtain integrability conditions of distributions. Then we give some curvature properties of skew semi invariant submanifold of para Kenmotsu space form. Finally we prove that a totally geodesic skew semi invariant submanifold of para Kenmotsu manifold is $\eta$-Einstein.

\section{Preliminaries}

Definition 1 Let $\overline{\mathrm{M}}^{(2 n+1)}$ differentiable manifold and $\varphi, \eta, \xi, g$ is $(1,1)$-tensor field, 1 -form, a vector field and a pseudo-Riemannian metric on $\bar{M}$, respectively. If for all $\mathrm{W}, \mathrm{Y} \in \Gamma(\mathrm{T} \overline{\mathrm{M}})$ following conditions are satifeied then $\overline{\mathrm{M}}$ called almost para contact metric manifold:

$\varphi^{2} \mathrm{~W}=\mu(\mathrm{W}-\eta(\mathrm{W}) \xi), \quad \eta(\xi)=1$

$\mathrm{g}(\varphi \mathrm{W}, \varphi \mathrm{Y})=-\mu(\mathrm{g}(\mathrm{W}, \mathrm{Y})-\varepsilon \eta(\mathrm{W}) \eta(\mathrm{Y}))$

where $\mu, \epsilon= \pm 1$. In addition, we have

$\varphi(\xi)=0, \eta \circ \varphi=0, \eta(W)=\varepsilon g(W, \xi)$.

By this definition $\bar{M}$ is an almost contact metric manifold if $\mu=-1$. For an almost contact metric manifold by setting $\varepsilon=1$ we get the signature of metric is equal to $2 p$, and by setting $\varepsilon=-1$, the signature of metric is equal to $2 p+1$. Also $\bar{M}$ is an almost paracontact metric manifold if $\mu=1$. In this case the signature of metric is equal to $n$ by setting $\varepsilon=1$, or $n+1$ by setting $\varepsilon=-1$ (Olszak, 2013).

Definition 2 An almost para contact metric manifold $\overline{\mathrm{M}}$ is normal if

$[\varphi, \varphi](\mathrm{W}, \mathrm{Y})-2 \mathrm{~d} \eta(\mathrm{W}, \mathrm{Y}) \xi=0$

for all $W, Y \in \Gamma(T \bar{M})$, where

$[\varphi, \varphi](\mathrm{W}, \mathrm{Y})=\varphi^{2}[\mathrm{~W}, \mathrm{Y}]+[\varphi \mathrm{W}, \varphi \mathrm{Y}]-$ $\varphi[\varphi \mathrm{W}, \mathrm{Y}]-\varphi[\mathrm{W}, \varphi \mathrm{Y}]($ Olszak, 2013).

Proposition 1 On an almost para contact metric manifold for any $\mathrm{W}, \mathrm{Y}, \mathrm{U} \in \Gamma(\mathrm{T} \overline{\mathrm{M}})$ we have

$$
\begin{aligned}
2 \mathrm{~g}\left(\left(\bar{\nabla}_{\mathrm{W}} \varphi\right) \mathrm{Y}, \mathrm{U}\right) & =3 \mathrm{~d} \Phi(\mathrm{W}, \varphi \mathrm{Y}, \varphi \mathrm{U}) \\
& -3 \mathrm{~d} \Phi(\mathrm{W}, \mathrm{Y}, \mathrm{U}) \\
& +\mathrm{g}(\mathrm{N}(\mathrm{Y}, \mathrm{U}), \varphi \mathrm{W}) \\
& +\mu \mathrm{N}^{2}(\mathrm{Y}, \mathrm{U}) \eta(\mathrm{W}) \\
& +2 \mu \mathrm{d} \eta(\varphi \mathrm{Y}, \mathrm{W}) \eta(\mathrm{U}) \\
& -2 \mu \mathrm{d} \eta(\varphi \mathrm{U}, \mathrm{W}) \eta(\mathrm{Y})
\end{aligned}
$$

where $\bar{\nabla}$ is Levi-Cevita connection on $\bar{M}$ and $\mathrm{N}^{2}(\mathrm{~W}, \mathrm{Y})=2 \mathrm{~d} \eta(\varphi \mathrm{W}, \mathrm{Y})-2 \mathrm{~d} \eta(\varphi \mathrm{Y}, \mathrm{W})$.

Definition 2 An almost para Kenmotsu manifold is an almost para contact metric manifold which has closed 1-form $\eta$ and $d \Phi=2 \eta \wedge \Phi$. If $\bar{M}$ is also normal then we call $\bar{M}$ is called a para Kenmotsu manifold.

Theorem $1 \mathrm{M}$ is para Kenmotsu manifold if and only if

$\left(\bar{\nabla}_{\mathrm{W}} \varphi\right) \mathrm{Y}=\mathrm{g}(\varphi \mathrm{W}, \mathrm{Y}) \xi-\eta(\mathrm{Y}) \varphi \mathrm{W}$

for all $\mathrm{W}, \mathrm{Y} \in \Gamma(\mathrm{T} \overline{\mathrm{M}})$.

Proof. Let $\bar{M}$ be a para Kenmotsu manifold. From Proposition 1, $\forall \mathrm{W}, \mathrm{Y} \in \Gamma(\mathrm{TM})$ we have

$$
\begin{aligned}
2 \mathrm{~g}\left(\left(\bar{\nabla}_{\mathrm{W}} \varphi\right) \mathrm{Y}, \mathrm{U}\right) & =3 \mathrm{~d} \Phi(\mathrm{W}, \varphi \mathrm{Y}, \varphi \mathrm{U}) \\
& -3 \mathrm{~d} \Phi(\mathrm{W}, \mathrm{Y}, \mathrm{U}))
\end{aligned}
$$

From the definition of second fundamental form we get 
$\mathrm{g}\left(\left(\bar{\nabla}_{\mathrm{W}} \varphi\right) \mathrm{Y}, \mathrm{U}\right)=-\eta(\mathrm{W}) \mathrm{g}\left(\varphi \mathrm{Y}, \varphi^{2} \mathrm{U}\right)+\eta(\mathrm{W}) \mathrm{g}(\mathrm{Y}, \varphi \mathrm{U})-\eta(\mathrm{Y}) \mathrm{g}(\mathrm{U}, \varphi \mathrm{W})-\eta(\mathrm{U}) \mathrm{g}(\mathrm{W}, \varphi \mathrm{Y})$.

Therefore from (1) and (2) we obtain (4).

Conversely using (4), we get $\varphi \bar{\nabla}_{\mathrm{W}} \xi=$ $\mathrm{g}(\varphi \mathrm{W}, \xi) \xi-\eta(\xi) \varphi \mathrm{W}$ and therefore $\bar{\nabla}_{\mathrm{W}} \xi=\varphi^{2} \mathrm{~W}$.

On the other hand for $\eta 1$-form we have

$\mathrm{d} \eta(\mathrm{W}, \mathrm{Y})=\frac{1}{2}\left\{\mathrm{~g}\left(\mathrm{Y},-\varphi^{2} \mathrm{~W}\right)-\mathrm{g}\left(\mathrm{W},-\varphi^{2} \mathrm{Y}\right)\right\}=0$

and this shows 1 -form $\eta$ is closed. In addition since

$3 \mathrm{~d} \Phi(\mathrm{W}, \mathrm{Y}, \mathrm{U})=\mathrm{g}(\mathrm{Y},(\bar{\nabla} \varphi) \mathrm{U})-\mathrm{g}\left(\mathrm{U},\left(\bar{\nabla}_{\mathrm{Y}} \varphi\right) \mathrm{W}\right)$ $-\mathrm{g}\left(\mathrm{W},\left(\bar{\nabla}_{\mathrm{U}} \varphi\right) \mathrm{Y}\right)$

and from (4), we have

$$
\begin{aligned}
& \begin{aligned}
3 \mathrm{~d} \Phi(\mathrm{W}, \mathrm{Y}, \mathrm{U}) & =\{\mathrm{g}(\varphi \mathrm{W}, \mathrm{U}) \mathrm{g}(\mathrm{Y}, \xi) \\
& -\eta(\mathrm{U}) \mathrm{g}(\mathrm{Y}, \varphi \mathrm{W}) \\
& -\mathrm{g}(\varphi \mathrm{Y}, \mathrm{U}) \mathrm{g}(\mathrm{X}, \xi) \\
+\eta(\mathrm{U}) \mathrm{g}(\mathrm{W}, \varphi \mathrm{Y}) & +\mathrm{g}(\varphi \mathrm{U}, \mathrm{Y}) \mathrm{g}(\mathrm{X}, \xi)- \\
\eta(\mathrm{Y}) \mathrm{g}(\mathrm{W}, \varphi \mathrm{U})\} & \\
= & 2\{\Phi(\mathrm{U}, \mathrm{W}) \eta(\mathrm{Y}) \\
& +\Phi(\mathrm{W}, \mathrm{Y}) \eta(\mathrm{U})+\Phi(\mathrm{Y}, \mathrm{U}) \eta(\mathrm{W})\}
\end{aligned}
\end{aligned}
$$

Thus we get $\mathrm{d} \Phi=2 \eta \wedge \Phi$. Moreover, the Nijenhuis tensor of $\varphi$ is

$$
\begin{aligned}
\mathrm{N}_{\varphi}(\mathrm{W}, \mathrm{Y})=\varphi( & -\{\mathrm{g}(\varphi \mathrm{W}, \mathrm{Y}) \xi-\eta(\mathrm{Y}) \varphi \mathrm{W}\}+\{\mathrm{g}(\varphi \mathrm{Y}, \mathrm{W}) \xi-\eta(\mathrm{W}) \varphi \mathrm{Y}\})+\left\{\mathrm{g}\left(\varphi^{2} \mathrm{~W}, \mathrm{Y}\right) \xi-\eta(\mathrm{Y}) \varphi^{2} \mathrm{~W}\right\} \\
& -\left\{\mathrm{g}\left(\varphi^{2} \mathrm{Y}, \mathrm{W}\right) \xi-\eta(\mathrm{W}) \varphi^{2} \mathrm{Y}\right\}=0
\end{aligned}
$$

Hence, $\bar{M}$ is normal. The proof is completed. By above theorem we get following corollary.

Corollary 1 For a para Kenmotsu manifold with $(\mathrm{M}, \varphi, \xi, \eta, \mathrm{g})$ and $\mathrm{W}, \mathrm{Y} \in \Gamma(\mathrm{T} \overline{\mathrm{M}})$ we have

$\bar{\nabla}_{\mathrm{W}} \xi=\varphi^{2} \mathrm{~W}$.

\section{Submanifolds of Para Kenmotsu Manifold}

Let $M^{n}$ be a submanfold of a para Kenmotsu manifold $\bar{M}^{(2 n+1)}$. The fundamental equations of the submanifold theory for $M$ are given by

$\bar{\nabla}_{W} Y=\nabla_{\mathrm{W}} \mathrm{Y}-\mathrm{h}(\mathrm{W}, \mathrm{Y})$ (Gauss Equation)

$\bar{\nabla}_{W} V=-\mathrm{A}_{V} \mathrm{~W}+\nabla_{\mathrm{W}}^{\frac{1}{\mathrm{~V}}} \mathrm{~V}$ (Weingarten Equaiton) (7)

where $W, Y \in \Gamma(T M)$ and $V \in \Gamma(T M)^{\perp}$. In this equations $h$ is denoting the second fundamental form, $\nabla^{\perp}$ is denoting the normal bundle connection and $A_{V}$ is shape operator associated with $V$. $A$ and $h$ related by

$$
g(h(W, Y), V)=g\left(\mathrm{~A}_{\mathrm{V}} W, Y\right) .
$$

The mean curvature tensor $H$ is defined by

$H=\frac{1}{m} \sum_{k=1}^{m} h\left(e_{k}, e_{k}\right)$

where $\left\{e_{1}, \ldots, e_{m}\right\}$ is a local orthonormal basis of $T M$.

For every tangent vector field $W$ on $M$ we can write

$\varphi W=T W+N W$ where $T W$ (resp. $N W$ ) denotes the tangential (resp. normal) component of $\varphi W$ and for every normal vector field $V$ we can state

$\varphi V=t V+n V$

where $t V$ in the tangental component of $\varphi V$ and $n V$ is the normal one.

Now, for later use, we establish some results for a submanifold para Kenmotsu manifold.

Proposition 2 On $\mathrm{M}^{\mathrm{n}}$ we have

$\left(\nabla_{\mathrm{W}} \mathrm{T}\right) \mathrm{Y}=\mathrm{A}_{\mathrm{NY}} \mathrm{W}+\mathrm{th}(\mathrm{W}, \mathrm{Y})+\mathrm{g}(\mathrm{TW}, \mathrm{Y}) \xi-$ $\eta(\mathrm{Y}) \mathrm{TW}$

$\left(\nabla_{\mathrm{W}} \mathrm{N}\right) \mathrm{Y}=\mathrm{nh}(\mathrm{W}, \mathrm{Y})-\mathrm{h}(\mathrm{W}, \mathrm{TY})-\eta(\mathrm{Y}) \mathrm{NW}(13)$

for all $\mathrm{W}, \mathrm{Y} \in \Gamma(\mathrm{TM})$

Proof. For any $\mathrm{W}, \mathrm{Y} \in \Gamma(\mathrm{TM})$ we have

$\left(\bar{\nabla}_{\mathrm{W}} \varphi\right) \mathrm{Y}=\bar{\nabla}_{\mathrm{W}} \varphi \mathrm{Y}-\varphi \bar{\nabla}_{\mathrm{W}} \mathrm{Y}$

Then, using (4), (6) and (7) we get

$\mathrm{g}(\mathrm{TW}+\mathrm{NW}, \mathrm{Y}) \xi-\eta(\mathrm{Y})(\mathrm{TW}+\mathrm{NW})$

$=\bar{\nabla}_{\mathrm{W}}(\mathrm{TY}+\mathrm{NY})-\varphi\left(\nabla_{\mathrm{W}} \mathrm{Y}\right.$

$+\mathrm{h}(\mathrm{W}, \mathrm{Y}))$

$=\nabla_{\mathrm{W}} \mathrm{TY}+\mathrm{h}(\mathrm{W}, \mathrm{TY})-\mathrm{A}_{\mathrm{NY}} \mathrm{W}+\nabla_{\mathrm{W}}^{\perp} \mathrm{NY}-\mathrm{T} \nabla_{\mathrm{W}} \mathrm{Y}$

$-N \nabla_{W} Y-\operatorname{th}(W, Y)-n h(W, Y)$

$=\left(\nabla_{\mathrm{W}} \mathrm{T}\right) \mathrm{Y}+\left(\nabla_{\mathrm{W}} \mathrm{N}\right) \mathrm{Y}+\mathrm{h}(\mathrm{W}, \mathrm{TY})$

$-A_{N Y} W-\operatorname{th}(W, Y)-\operatorname{nh}(W, Y)$

and thus we obtain

$$
\begin{aligned}
&\left(\nabla_{\mathrm{W}} \mathrm{T}\right) \mathrm{Y}+\left(\nabla_{\mathrm{W}} \mathrm{N}\right) \mathrm{Y} \\
&=\mathrm{g}(\mathrm{TW}+\mathrm{NW}, \mathrm{Y}) \xi-\eta(\mathrm{Y}) \mathrm{TW} \\
&-\eta(\mathrm{Y}) \mathrm{NW} \\
&-\mathrm{h}(\mathrm{W}, \mathrm{TY})+\mathrm{A}_{\mathrm{NY}} \mathrm{W}+\mathrm{th}(\mathrm{W}, \mathrm{Y})-\mathrm{nh}(\mathrm{W}, \mathrm{Y}) .
\end{aligned}
$$

By cosider tangent and normal components in this equation, (12) and (13) is obtanied. 


\section{Skew Invariant Submanifolds of Para Kenmotsu Manifold}

In 1990, Ronsse defined skew semi invariant submanifold of a contact metric manifold. In this chapter we use this definition for para Kenmotsu manifolds.

Definition 3 Let $\left(\overline{\mathrm{M}}^{(2 n+1)}, \varphi, \xi, \mathrm{g}\right)$ be a para Kenmotsu manifold and $\mathrm{M}^{\mathrm{n}}$ be a submanifold of $\overline{\mathrm{M}}$ which is tangent to $\xi$. For s distinct functions $\mu_{1}, \mu_{2}, \ldots, \mu_{\mathrm{s}}$ defined on $\mathrm{M}$ with values in the open interval $(0,1)$ such that TM is decomposed as T invariant and mutually orthogonal differentiable distributions given by

$\mathrm{TM}=\mathrm{D}^{0} \oplus \mathrm{D}^{1} \oplus \mathrm{D}^{\mu_{1}} \oplus \ldots \oplus \mathrm{D}^{\mu_{\mathrm{s}}} \oplus\{\xi\}$

\section{Example 1}

The para Kenmotsu structure on canonic contact manifold $\left(\mathbb{R}^{2 \mathrm{n}+1}, \varphi, \xi, \eta, \mathrm{g}\right)$ is given by

$$
\begin{aligned}
& \varphi\left(\mathrm{U}_{1}, \ldots, \mathrm{U}_{\mathrm{n}}, \mathrm{V}_{1}, \ldots, \mathrm{V}_{\mathrm{n}}, \xi\right)=\left(\mathrm{V}_{1}, \ldots, \mathrm{V}_{\mathrm{n}},-\mathrm{U}_{1}, \ldots,-\mathrm{U}_{\mathrm{n}}\right) \\
& \xi=\frac{\partial}{\partial z}, \quad \eta=d z \\
& g=e^{-2 z} \sum_{i=1}^{n}\left[d x_{i} \otimes d x_{i}+d y_{i} \otimes d y_{i}\right]-\eta \otimes \eta
\end{aligned}
$$$$
\begin{gathered}
\mathrm{e}_{1}=\frac{\partial}{\partial \mathrm{x}_{1}}, \quad \mathrm{e}_{2}=\frac{\partial}{\partial \mathrm{y}_{1}}, \quad \mathrm{e}_{3}=\frac{\partial}{\partial \mathrm{x}_{3}}, \quad \mathrm{e}_{4}=\frac{\partial}{\partial \mathrm{y}_{2}}, \mathrm{e}_{5}=\frac{\partial}{\partial \mathrm{x}_{4}} \\
\mathrm{e}_{6}=\operatorname{sinw} \frac{\partial}{\partial \mathrm{y}_{3}}+\operatorname{cosw} \frac{\partial}{\partial \mathrm{y}_{4}}, \quad \mathrm{e}_{7}=\xi=\frac{\partial}{\partial \mathrm{z}}
\end{gathered}
$$

Then we have a local frame of TM by

and

$$
\mathrm{e}_{1}{ }^{*}=\frac{\partial}{\partial \mathrm{x}_{2}}, \mathrm{e}_{2}^{*}=\frac{\partial}{\partial \mathrm{y}_{3}}
$$

from a basis of $\mathrm{T}^{\perp} \mathrm{M}$. We determine $\mathrm{D}^{0}=$ $\operatorname{Sp}\left\{\mathrm{e}_{1}, \mathrm{e}_{2}\right\}, \mathrm{D}^{1}=\operatorname{Sp}\left\{\mathrm{e}_{3}, \mathrm{e}_{4}\right\}$ and $\mathrm{D}^{\mu}=\operatorname{Sp}\left\{\mathrm{e}_{5}, \mathrm{e}_{6}\right\}$. Thus we get $\mathrm{TM}=\mathrm{D}^{0} \oplus \mathrm{D}^{1} \oplus \mathrm{D}^{\mu}$ and $\mathrm{M}$ is a skew semi invariant submanifold of $\mathbb{R}^{9}$.

Now, let $\mathrm{M}^{\mathrm{n}}$ be an skew semi invariant submanifold of para Kenmotsu manifold $\bar{M}$. The projection morphisms of TM to the distributions $\mathrm{D}^{0}, \mathrm{D}^{1}$ and $\mathrm{D}^{\mu}$ are denoted respectively by $\mathrm{P}_{0}, \mathrm{P}_{1}$ and $\mathrm{P}_{2}$. Then for each $\mathrm{W} \in \Gamma(\mathrm{TM})$ we have $\mathrm{W}=\mathrm{P}_{0} \mathrm{~W}+\mathrm{P}_{1} \mathrm{~W}+\mathrm{P}_{2} \mathrm{~W}+\eta(\mathrm{W}) \xi$.

Thus from (10) we get $\mathrm{TW}=\mathrm{TP}_{0} \mathrm{~W}+\mathrm{TP}_{2} \mathrm{~W}$ and $\mathrm{NW}=\mathrm{NP}_{1} \mathrm{~W}+\mathrm{NP}_{2} \mathrm{~W}$.

By using (5), (6) and (14) and several computations we obtaion following propositions.

Proposition 3 On $M$ for $Y \in \Gamma(\mathrm{TM})$ we have

$g\left(P_{0} W, Y\right)=g\left(W, P_{0} Y\right)$, for any $W, Y \in \Gamma\left(D^{0}\right)$

$g\left(P_{1} W, Y\right)=g\left(W, P_{1} Y\right), \quad$ for any $W, Y \in \Gamma\left(D^{1}\right)$

$g\left(P_{2} W, Y\right)=g\left(W, P_{2} Y\right)$, for any $W, Y \in \Gamma\left(D^{\mu}\right)$

$g\left(P_{i} W, Y\right)=g\left(W, P_{j} Y\right)$, for any $W, Y \in \Gamma(T M), i \neq j$ and $i, j \in\{0,1,2\}$

$\nabla_{\mathrm{W}} \xi=\mathrm{P}_{0} \mathrm{~W}, \mathrm{~h}(\mathrm{~W}, \xi)=0$ for any $\mathrm{W} \in \Gamma\left(\mathrm{D}^{0}\right)$

$\nabla_{\mathrm{W}} \xi=0, \mathrm{~h}(\mathrm{~W}, \xi)=\mathrm{P}_{1} \mathrm{~W}$ for any $\mathrm{W} \in \Gamma\left(\mathrm{D}^{1}\right)$

$\nabla_{\mathrm{W}} \xi=\varphi \mathrm{TP}_{2} \mathrm{~W}, \mathrm{~h}(\mathrm{~W}, \xi)=\varphi \mathrm{NP}_{2} \mathrm{~W}$ for any $\mathrm{W} \in \Gamma\left(\mathrm{D}^{\mu}\right)$ 
Theorem 2 Let $\mathrm{M}^{\mathrm{n}}$ be skew semi invariant submanifold of para Kenmotsu manifold $\bar{M}$. The distrbution $\mathrm{D}^{0}$ is not integrable.

Proof For all $\mathrm{W}, \mathrm{Y} \in \Gamma\left(\mathrm{D}^{0}\right)$ and from equation (19)

$$
\begin{aligned}
\mathrm{g}([\mathrm{W}, \mathrm{Y}], \xi) & =\mathrm{g}\left(\nabla_{\mathrm{W}} \mathrm{Y}, \xi\right)-\mathrm{g}\left(\nabla_{\mathrm{Y}} \mathrm{W}, \xi\right) \\
& =-\mathrm{g}\left(\mathrm{Y}, \nabla_{\mathrm{W}} \xi\right)+\mathrm{g}\left(\mathrm{W}, \nabla_{\mathrm{Y}} \xi\right) \\
& =\mathrm{g}\left(\mathrm{Y}, \mathrm{P}_{0} \mathrm{~W}\right)-\mathrm{g}\left(\mathrm{W}, \mathrm{P}_{0} \mathrm{Y}\right) \\
& =2 \mathrm{~g}\left(\mathrm{~W}, \mathrm{P}_{0} \mathrm{Y}\right) .
\end{aligned}
$$

Thus $\mathrm{D}^{0}$ is integrable if and only if $\mathrm{g}\left(\mathrm{W}, \mathrm{P}_{0} \mathrm{Y}\right)=$ 0 . From (15) the proof is completed.

Theorem 3 Let $\mathrm{M}^{\mathrm{n}}$ be skew semi invariant submanifold of para Kenmotsu manifold $\overline{\mathrm{M}}$. The distribution $\mathrm{D}^{1}$ is always integrable.

Proof for all $\mathrm{W}, \mathrm{Y} \in \Gamma\left(\mathrm{D}^{1}\right)$, from (20)

Proof For all $\left.W, Y \in \Gamma\left(D^{0} \oplus \xi\right\}\right)$

$$
\begin{aligned}
\varphi([\mathrm{W}, \mathrm{Y}])= & \varphi \bar{\nabla}_{\mathrm{W}} \mathrm{Y}-\varphi \bar{\nabla}_{\mathrm{Y}} \mathrm{W} \\
= & \bar{\nabla}_{\mathrm{W}} \varphi \mathrm{Y}-\left(\bar{\nabla}_{\mathrm{W}} \varphi\right) \mathrm{Y}-\bar{\nabla}_{\mathrm{Y}} \varphi \mathrm{W}-\left(\bar{\nabla}_{\mathrm{Y}} \varphi\right) \mathrm{W} \\
= & \nabla_{\mathrm{W}} \varphi \mathrm{Y}-\mathrm{h}(\mathrm{W}, \varphi \mathrm{Y})-\mathrm{g}(\varphi \mathrm{W}, \mathrm{Y}) \xi-\eta(\mathrm{Y}) \varphi \mathrm{W} \\
& \quad+\nabla_{\mathrm{Y}} \varphi \mathrm{W}-\mathrm{h}(\mathrm{Y}, \varphi \mathrm{W})-\mathrm{g}(\varphi \mathrm{Y}, \mathrm{W}) \xi-\eta(\mathrm{W}) \varphi \mathrm{Y} .
\end{aligned}
$$

$$
\mathrm{g}([\mathrm{Y}, \mathrm{W}], \xi)=\mathrm{g}\left(\nabla_{\mathrm{Y}} \mathrm{W}, \xi\right)-\mathrm{g}\left(\nabla_{\mathrm{W}} \mathrm{Y}, \xi\right)=
$$$$
-\mathrm{g}\left(\mathrm{W}, \nabla_{\mathrm{Y}} \xi\right)+\mathrm{g}\left(\mathrm{Y}, \nabla_{\mathrm{W}} \xi\right)=0 \text {. }
$$

Theorem 4 Let $\mathrm{M}^{\mathrm{n}}$ be skew semi invariant submanifold of para Kenmotsu manifold $\bar{M}$. The distribution $D^{\mu}$ is always integrable.

Proof For all $\mathrm{W}, \mathrm{Y} \in \Gamma\left(\mathrm{D}^{\mu}\right)$, from equation (21)

$$
\begin{aligned}
\mathrm{g}([\mathrm{W}, \mathrm{Y}], \xi) & =-\mathrm{g}\left(\mathrm{Y}, \nabla_{\mathrm{W}} \xi\right)+\mathrm{g}\left(\mathrm{W}, \nabla_{\mathrm{Y}} \xi\right) \\
& =-\mathrm{g}\left(\mathrm{Y}, \varphi \mathrm{TP}_{2} \mathrm{~W}\right)+\mathrm{g}\left(\mathrm{W}, \varphi \mathrm{TP}_{2} \mathrm{Y}\right) \\
& =\mathrm{g}\left(\varphi \mathrm{P}_{2} \mathrm{Y}, \mathrm{TP}_{2} \mathrm{~W}\right)-\mathrm{g}\left(\varphi \mathrm{P}_{2} \mathrm{~W}, \mathrm{TP}_{2} \mathrm{Y}\right) \\
& =\mathrm{g}\left(\mathrm{TP}_{2} \mathrm{Y}, \mathrm{TP}_{2} \mathrm{~W}\right)-\mathrm{g}\left(\mathrm{TP}_{2} \mathrm{~W}, \mathrm{TP}_{2} \mathrm{Y}\right) \\
& =0 .
\end{aligned}
$$

Theorem 5 Let $M$ be skew semi invariant submanifold of para Kenmotsu manifold $\overline{\mathrm{M}}$. The distrbution $\mathrm{D}^{0} \oplus\{\xi\}$ is always integrable if and only if $h(\mathrm{~W}, \varphi \mathrm{Y})=\mathrm{h}(\mathrm{Y}, \varphi \mathrm{W})$.
Then we give $\left.[\mathrm{W}, \mathrm{Y}] \in \Gamma\left(\mathrm{D}^{0} \oplus \xi\right\}\right)$ if and only if $\mathrm{h}(\mathrm{W}, \varphi \mathrm{Y})=\mathrm{h}(\mathrm{Y}, \varphi \mathrm{W})$, where $\varphi([\mathrm{W}, \mathrm{Y}])$ shows the component of $\nabla_{\mathrm{W}} \mathrm{Y}$ from ortogonal complementary distribution of $\mathrm{D}^{0} \oplus\{\xi\}$ in $\mathrm{M}$.

Corollary 2 Let $M$ be skew semi invariant submanifold of para Kenmotsu manifold $\bar{M}$. The distribution $\mathrm{D}^{1} \oplus\{\xi\}$ is always integrable if and only if $A_{\varphi \mathrm{Y}} \mathrm{W}=\mathrm{A}_{\varphi \mathrm{W}} \mathrm{Y}$.

Theorem 6 Let $M$ be skew semi invariant submanifold of para Kenmotsu manifold $\bar{M}$. The distribution $\mathrm{D}^{0} \oplus \mathrm{D}^{1}$ is not integrable.

Proof For all $\mathrm{W}, \mathrm{Y} \in \Gamma\left(\mathrm{D}^{0} \oplus \mathrm{D}^{1}\right)$

$$
\begin{aligned}
\mathrm{g}([\mathrm{W}, \mathrm{Y}], \xi) & =-\mathrm{g}\left(\mathrm{Y}, \nabla_{\mathrm{W}} \xi\right)+\mathrm{g}\left(\mathrm{W}, \nabla_{\mathrm{Y}} \xi\right) \\
& =\mathrm{g}\left(\mathrm{Y}, \mathrm{P}_{0} \mathrm{~W}+\mathrm{P}_{1} \mathrm{~W}\right)-\mathrm{g}\left(\mathrm{W}, \mathrm{P}_{0} \mathrm{Y}+\right.
\end{aligned}
$$$$
\left.\mathrm{P}_{1} \mathrm{Y}\right)
$$

$$
=2 g\left(Y, P_{0} W\right)-2 g\left(W, P_{1} Y\right)
$$

Thus $\mathrm{D}^{0} \oplus \mathrm{D}^{1}$ is integrable if and only if $g\left(Y, P_{0} W\right)=g\left(W, P_{1} Y\right)$. From (18) the proof is completed.

Corollary 3 Let $M$ be skew semi invariant submanifold of para Kenmotsu manifold $\bar{M}$. The distribution $\mathrm{D}^{0} \oplus \mathrm{D}^{\mu}$ and $\mathrm{D}^{1} \oplus \mathrm{D}^{\mu}$ is not integrable.

\section{Skew Invariant Submanifolds of Para Kenmotsu Space Form}

One of the special classes of contact manifolds is contact space form which has constant $\varphi-$ sectional curvature. In this section we study on skew semi invariant submanifolds of para Kenmotsu space forms. The Riemannian curvature of a submanifold of para Kenmotsu space form $\bar{M}$ is given by

$$
\begin{aligned}
\mathrm{R}(\mathrm{W}, \mathrm{Y}, \mathrm{U}, \mathrm{V})=\frac{\mathrm{c}+3}{4}(\mathrm{~g}(\mathrm{Y}, \mathrm{U}) \mathrm{g}(\mathrm{W}, \mathrm{V})-\mathrm{g}(\mathrm{W}, \mathrm{U}) \mathrm{g}(\mathrm{Y}, \mathrm{V})) \\
+\frac{\mathrm{c}-1}{4}(\mathrm{~g}(\mathrm{Y}, \varphi \mathrm{U}) \mathrm{g}(\varphi \mathrm{W}, \mathrm{V})-\mathrm{g}(\mathrm{W}, \varphi \mathrm{U}) \mathrm{g}(\varphi \mathrm{Y}, \mathrm{V})-2 \mathrm{~g}(\mathrm{~W}, \varphi \mathrm{Y}) \mathrm{g}(\varphi \mathrm{U}, \mathrm{V}) \\
\quad+\mathrm{g}(\mathrm{W}, \mathrm{U}) \eta(\mathrm{Y}) \eta(\mathrm{V})-\mathrm{g}(\mathrm{Y}, \mathrm{U}) \eta(\mathrm{W}) \eta(\mathrm{V})+\mathrm{g}(\mathrm{Y}, \mathrm{V}) \eta(\mathrm{W}) \eta(\mathrm{U}) \\
\quad-\mathrm{g}(\mathrm{W}, \mathrm{V}) \eta(\mathrm{Y}) \eta(\mathrm{U}))+\mathrm{g}(\mathrm{h}(\mathrm{W}, \mathrm{U}), \mathrm{h}(\mathrm{Y}, \mathrm{V}))-\mathrm{g}(\mathrm{h}(\mathrm{Y}, \mathrm{U}), \mathrm{h}(\mathrm{W}, \mathrm{V})) .
\end{aligned}
$$

for all $\mathrm{W}, \mathrm{Y}, \mathrm{U}, \mathrm{V} \in \Gamma(\mathrm{TM})$, where $\mathrm{c}$ is constant $\varphi$-sectional curvature of $\overline{\mathrm{M}}$. Now, we define a local field of orthonormal frames $\mathrm{D}^{0}=\operatorname{sp}\left\{\mathrm{e}_{1}, \ldots, \mathrm{e}_{2 \mathrm{p}}\right\}, \mathrm{D}^{1}=\operatorname{sp}\left\{\mathrm{e}_{2 \mathrm{p}+1}, \ldots, \mathrm{e}_{2 \mathrm{q}}\right\}, \mathrm{D}^{2}=\operatorname{sp}\left\{\mathrm{e}_{2 \mathrm{q}+1}, \ldots \mathrm{e}_{2 \mathrm{r}}, \mathrm{e}_{2 \mathrm{r}+1}\right\}$,

$\xi=\operatorname{sp}\left\{\mathrm{e}_{2 \mathrm{r}+1}\right\}$

where $\operatorname{dim} D^{0}=2 p, \operatorname{dim} D^{1}=2 q$ and $\operatorname{dim} D^{2}=$ 2r. $\left\{\mathrm{e}_{1}, \ldots, \mathrm{e}_{2 \mathrm{p}}, \mathrm{e}_{2 \mathrm{p}+1}, \ldots, \mathrm{e}_{2 \mathrm{q}}, \mathrm{e}_{2 \mathrm{q}+1}, \ldots \mathrm{e}_{2 \mathrm{r}}, \mathrm{e}_{2 \mathrm{r}+1}\right\}$ on skew semi invariant submanifold $M$. Then we have

Corollary 4 For a skew semi invariant submanifold of para Kenmotsu space form we have 


$$
\begin{aligned}
\mathrm{R}(\mathrm{W}, \mathrm{Y}, \mathrm{U}, \mathrm{V})= & \frac{\mathrm{c}+3}{4}(\mathrm{~g}(\mathrm{Y}, \mathrm{U}) \mathrm{g}(\mathrm{W}, \mathrm{V})-\mathrm{g}(\mathrm{W}, \mathrm{U}) \mathrm{g}(\mathrm{Y}, \mathrm{V})) \\
& +\frac{\mathrm{c}-1}{4}(\mathrm{~g}(\mathrm{Y}, \varphi \mathrm{U}) \mathrm{g}(\varphi \mathrm{W}, \mathrm{V})-\mathrm{g}(\mathrm{W}, \varphi \mathrm{U}) \mathrm{g}(\varphi \mathrm{Y}, \mathrm{V})-2 \mathrm{~g}(\mathrm{~W}, \varphi \mathrm{Y}) \mathrm{g}(\varphi \mathrm{U}, \mathrm{V}) \\
& +\mathrm{g}(\mathrm{h}(\mathrm{W}, \mathrm{U}), \mathrm{h}(\mathrm{W}, \mathrm{V}))-\mathrm{g}(\mathrm{h}(\mathrm{Y}, \mathrm{U}), \mathrm{h}(\mathrm{W}, \mathrm{V}))
\end{aligned}
$$

for all $\mathrm{W}, \mathrm{Y}, \mathrm{U}, \mathrm{V} \in \Gamma\left(\mathrm{D}^{0}\right)$.

Proof For all $\mathrm{W} \epsilon \Gamma\left(D^{0}\right)$, from $\eta(W)=0$. Using (22), the desired equality is obtained.
Theorem 7 Let $M$ be skew semi invariant submanifold of para Kenmotsu space form $\bar{M}$ and $\mathrm{D}^{1}$ be totally geodesic. Then $\mathrm{M}$ is flat if and only if $\mathrm{c}=-3$.

Proof For all $\mathrm{W}, \mathrm{Y}, \mathrm{U}, \mathrm{V} \in \Gamma\left(\mathrm{D}^{1}\right)$ from (22) we have

$\mathrm{R}(\mathrm{W}, \mathrm{Y}, \mathrm{U}, \mathrm{V})=\frac{\mathrm{c}+3}{4}(\mathrm{~g}(\mathrm{Y}, \mathrm{U}) \mathrm{g}(\mathrm{W}, \mathrm{V})-\mathrm{g}(\mathrm{W}, \mathrm{U}) \mathrm{g}(\mathrm{Y}, \mathrm{V}))+\mathrm{g}(\mathrm{h}(\mathrm{W}, \mathrm{U}), \mathrm{h}(\mathrm{Y}, \mathrm{V}))-\mathrm{g}(\mathrm{h}(\mathrm{Y}, \mathrm{U}), \mathrm{h}(\mathrm{W}, \mathrm{V}))$.

Since $\mathrm{D}^{1}$ be totally geodesic then $\mathrm{h}=0$ and so

$R(W, Y, U, V)=\frac{c+3}{4}(g(Y, U) g(W, V)-g(W, U) g(Y, V))$.

Thus $\mathrm{M}$ is flat if and only if $\mathrm{c}=-3$.

Theorem 8 Let $\mathrm{M}$ be a skew semi invariant submanifold of para Kenmotsu space form $\bar{M}$. If $\mathrm{M}$ is totally geodesic, then it is $\eta$-Einstein.
Proof Let M be totally geodesic skew semi invariant submanifold of para Kenmotsu space form $\overline{\mathrm{M}}$. For all $\mathrm{W}, \mathrm{Y} \in \Gamma(\mathrm{TM})$ by using (22) the Ricci tensor is

$$
\begin{aligned}
& S(W, Y)= \sum_{i=1}^{2 p} R\left(W, E_{i}, E_{i}, Y\right)+\sum_{j=2 p+1}^{2 q} R\left(W, E_{j}, E_{j}, Y\right)+\sum_{k=2 q+1}^{2 r} R\left(W, E_{k}, E_{k}, Y\right)+R(W, \xi, \xi, Y) \\
&=\left(\frac{c+3}{4}(2 p-1)-3 \frac{c-1}{4}\right) g(Y, U)+\sum_{i=1}^{2 p}\left\{g\left(h(W, Y), h\left(E_{i}, E_{i}\right)\right)-g\left(h\left(E_{i}, Y\right), h\left(W, E_{i}\right)\right)\right\} \\
& \quad+\left(\frac{c+3}{4}(2 q-1)-3 \frac{c-1}{4}\right) g(Y, U) \\
&+\sum_{j=2 p+1}^{2 q}\left\{g\left(h(W, Y), h\left(E_{j}, E_{j}\right)\right)-g\left(h\left(E_{j}, Y\right), h\left(W, E_{j}\right)\right)\right\} \\
& \quad+\left(\frac{c+3}{4}(2 r-1)-3 \frac{c-1}{4} \mu^{2}\right) g(Y, U)+\sum_{k=2 q+1}^{2 r}\left\{g\left(h(W, Y), h\left(E_{k}, E_{k}\right)\right)\right. \\
&\left.-g\left(h\left(E_{k}, Y\right), h\left(W, E_{k}\right)\right)\right\}+g(W, Y)-\eta(W) \eta(Y) .
\end{aligned}
$$

thus since $\mathrm{h}=0$ we get

$S(W, Y)=\left(\frac{c+3}{4}(2 p+2 q+2 r-3)-3 \frac{c-1}{4}\left(2+\mu^{2}\right)+1\right) g(W, Y)-\eta(W) \eta(Y)$.

Corollary 5 Let $M$ be an skew semi invariant submanifold of para Kenmotsu space form $\bar{M}$. Then the scalar curvature $\tau$ of $\mathrm{M}$ is given by

$$
\begin{aligned}
& \tau=\left(\frac{c+3}{4}(2 p+2 q+2 r-3)-3 \frac{c-1}{4}\left(2+\cos ^{2} \theta\right)+1\right)(2 p+2 q+2 r+1)-1 \\
& +\frac{1}{(2 p+2 q+2 r+1)^{2}}\|H\|^{2}+\|h\|^{2} .
\end{aligned}
$$

\section{Acknowledgements}

This paper is supported by Amasya University research project (FMB-BAP 17-0284)

\section{References}

Bejancu, A. and Papaghiuc, N., 1984. Almost Semi Invariant Submanifolds of a Sasakian 
Manifold. Bulletin Mathematique de la Societe des Sciences, 28(76), 13-30.

Cruceanu, V., Fortuny, P. and Gadea, P.M., 1996. A survey on paracomplex geometry. Rocky Mountain Journal of Mathematics, 26(1), 83-115.

Kaneyuki, S. and Willams, F.L., 1985. Almost paracontact and parahodge structures on manifolds. Nagoya Mathematical Journal, 99, 173-187.

Lui, X. and Shao, F.M., 1999. Skew Semi Invariant Submanifolds of a Locally Product Manifold. Portugaliae Mathematica, 56, 319-327.

Olszak, Z., 2013. The Schouten-Van Kampen Affine Connection Adapted to an Almost
Para Contact Metric Structure. Publications De L'Institut Mathematique, 94(108), 3142.

Ronsse, G.S., 1990. Generic and Skew CRSubmanifolds of a Kaehler Manifold. Bulletin of the Institute Mathematics Academia Sinica, 18, 127-141.

Şahin, B., 2010. Skew CR-waped product submanifolds of a Kaehler Manifolds. Mathematical Communications, 15, 189204

Zamkovoy, S. 2009, Canonical connections on para contact manifolds. Annals of Global Analisis and Geometry, 36(1), 37-60. 